\title{
Mesenteric desmoid tumor: Differential diagnosis with computed tomography
}

\author{
TIMOTHY DUNN, D.O. \\ Langhorne, Pennsylvania \\ RUSSELL GOLKOW, M.D. \\ Stratford, New Jersey
}

\begin{abstract}
Desmoid tumors represent a rare cause of mesenteric masses. They are sometimes found in association with Gardner's syndrome. A defect in growth regulation of connective tissue has been suggested as a basic cause, with trauma, sex hormones, and pregnancy as precipitating factors. Considered a benign process, they may produce symptoms through pressure on or even obstruction of adjacent organs or vascular structures. In addition, there is a high recurrence rate following surgical removal. In the present casethat of a 33-year-old pregnant patientmesenteric desmoid tumor was responsible for gastric outlet obstruction. As this case demonstrated, computed tomography plays an important role in the evaluation of patients with mesenteric masses, by providing valuable information concerning the location, density, and relation of the mass to contiguous structures.
\end{abstract}

Mesenteric desmoid tumors are rare fibroblastic lesions. The computed tomography (CT) appearance of mesenteric desmoids has not been reported widely, ${ }^{1-4}$ although CT has a valuable role to play in the differential diagnosis of mesenteric masses. We report a case of mesenteric desmoid tumor in a pregnant woman that resulted in gastric outlet obstruction.

\section{Report of case}

A 33-year-old, pregnant, white woman was admitted to Kennedy Memorial Hospitals-University Medical Center for diffuse, upper abdominal pain associated with nausea and vomiting. Her symptoms had begun rather abruptly 2 weeks prior to admission and had steadily increased in severity. The clinical history and a subsequent ultrasonographic study confirmed a viable intra- uterine pregnancy of approximately 38 weeks' gestation. On ultrasonography, there was no evidence of a uterine or adnexal mass; likewise, the gallbladder appeared normal. There was no history of Crohn's disease, biliary disease, or alcohol abuse. The serum amylase value was 130 Somogyi units (normal 20-200 Somogyi units).

Because of a sudden fall in the patient's hemoglobin value (from 12.6 to $10.5 \mathrm{gm}$./dl.) and the increasing severity of the patient's symptoms, induction of labor was performed, and a viable male infant was delivered without complications. Following delivery, a large upper abdominal mass could be palpated. Ultrasonography revealed normal spleen, liver, and kidneys, but it demonstrated a poorly defined, hyperechoic mass medial to the spleen. An abdominal CT scan with intravenous contrast medium demonstrated a $10 \times 7.5 \mathrm{~cm}$. mass in the upper anterior part of the abdomen, slightly to the left of midline, adjacent to the gastric antrum, and insinuating itself into the adjacent omentum and mesentery (Figs. 1 and 2). The mass was fairly homogeneous, with a density measuring 22 CT units, and appeared to be reasonably well demarcated from adjacent structures.

Development of symptoms of gastric outlet obstruction necessitated the placement of a nasogastric tube. For this reason and because of the sudden fall in the patient's hemoglobin value, the most likely diagnosis appeared to be mesenteric hematoma causing gastric outlet obstruction. Intraabdominal abscess was considered to be a less likely diagnosis because of the lack of clinical signs of sepsis. Hemorrhage within a uterine leiomyoma was excluded as a cause for the patient's symptoms by the original pelvic ultrasonographic examination, which showed no masses within or adjacent to the uterus, and by the postpartum abdominal CT scan, which clearly demonstrated that the mass arose from within the mesentery rather than from the pelvis.

At laparotomy, an indurated, focally hemorrhagic, elongated mass of adipose tissue, $10 \times 7 \times 3 \mathrm{~cm}$., was found to involve the middle half of the transverse mesocolon. The stomach was very adherent to the mass. The remainder of the abdominal exploration was negative.

The pathologic specimen was a mass of adipose tissue with extensive necrosis and a central cystic cavity. Extensive liquefaction and focal coagulation necrosis were noted throughout the specimen. There were areas of fibrous tissue proliferation with atypical reparative cells and foamy macrophages. The final pathologic impression 


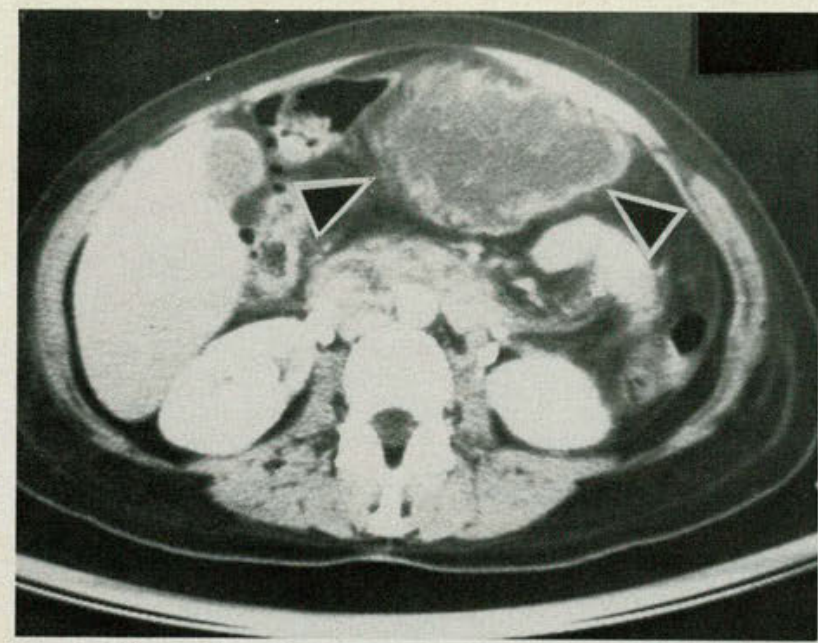

Fig. 1. CT scan demonstrates a mass (arrowheads) in the anterior portion of the abdomen slightly to the left of midline.

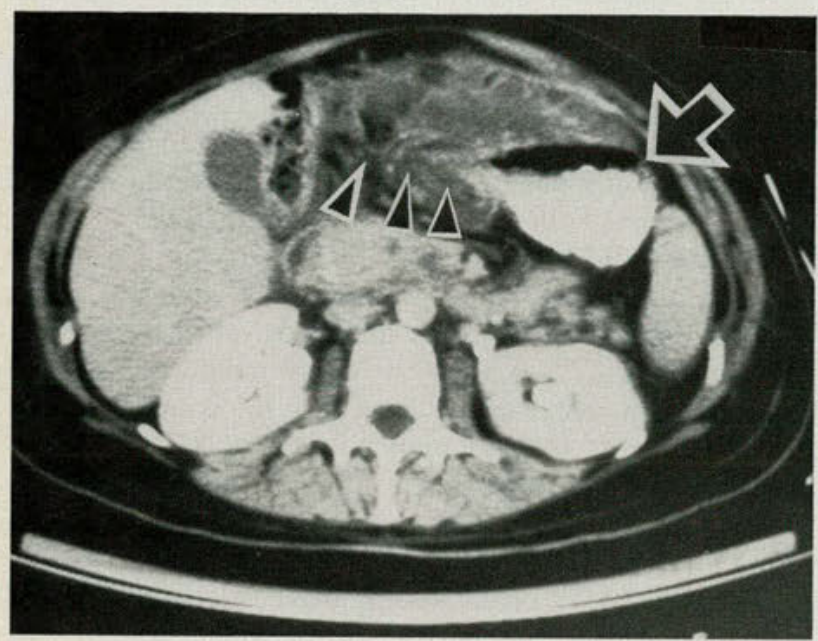

Fig. 2. CT scan slightly caudad to that in Figure 1 demonstrates insinuation of the mass into the mesentery (arrowheads). The contrast-filled stomach is indicated by the arrow.

was that of a benign process characterized by fibrous tissue proliferation and fat necrosis of the mesentery.

The patient's clinical status was much improved postoperatively, but she was subsequently lost to followup.

\section{Review of the literature}

Desmoid tumors are rare. They arise in the anterior abdominal wall from the rectus sheath more often than in the mesentery. 5,6 They are more common in women than in men, and most of the patients are between the ages of 20 and 40 years. ${ }^{6,7}$

The cause of fibromatosis is not known. Desmoid tumors have been found in association with Gardner's syndrome, ${ }^{8,9}$ which is due to an autosomal dominant gene. A history of surgical or other trauma at the site of the tumor has been reported in some but not all cases. ${ }^{10} \mathrm{~A}$ Finnish study ${ }^{11}$ has shown an association with surgical trauma in ab- dominal but not extraabdominal desmoid tumors.

Endocrine factors also have been suggested, with desmoid tumors having been induced experimentally by estrogen administration ${ }^{12}$ as well as having been responsive to testosterone therapy. ${ }^{13}$ Fertile women with desmoid tumor studied by Häryr and coworkers $^{11}$ had a hormonal imbalance consisting of estrogen predominance over progesterone. Growth rate of the tumor was much faster in this group and in menopausal women than in girls, males, or postmenopausal women. X-ray skeletal screening of these patients also revealed multiple minor bone malformations in up to 80 percent of patients. ${ }^{11}$ As a result, these Finnish investigators ${ }^{14}$ suggested that desmoid tumor is basically caused by an inherited defect in connective tissue formation. They believe that while trauma may be a precipitating factor in some cases, growth rate is mainly under the influence of steroid sex hormones.

The fibrous tissue that constitutes desmoid tumors is histologically normal throughout development and disintegration. They never display anaplasia, nor do they ever metastasize. However, they are highly infiltrative and thus have a significant incidence of recurrence (50.2 percent) after surgical removal. ${ }^{15}$

The histologic features of desmoid tumors have been described by Suarez and Hall ${ }^{10}$ and by Reitamo and coworkers. ${ }^{6}$ The characteristics described by Reitamo ${ }^{6}$ are as follows: proliferation of well-differentiated fibroblasts; infiltrative patterns of growth; the presence of variable (usually abundant) amounts of collagen between the proliferating cells; and lack of cytologic features of malignancy. Suarez and Hall ${ }^{10}$ noted similar features and theorized that the basic defect in desmoid tumors occurs at the level of the endoplasmic reticulum of the myofibroblast. Whereas in the normal fibroblast there is an inhibitor which prevents premature crosslinking of the tropocollagen molecule, this substance may fail to act in the fibromatosis, allowing collagen to complete its organization in intracytoplasmic fibers. They also state that while pregnancy has been implicated in the development of abdominal wall fibromatosis, the same relationship has not been noted in the intra-abdominal fibromatosis. The present case reports an unusual case of desmoid tumor in pregnancy in which the site of involvement was intraabdominal rather than in the abdominal wall.

Desmoid tumors may cause no symptoms. However, they may cause problems ranging from local pressure to abdominal obstruction or vascular compromise. ${ }^{1}$ In our patient, symptoms were related to gastric outlet obstruction caused by ad- 
herence and compression of the tumor to the gastric antrum.

Many recent articles show the diversity of conditions associated with mesenteric masses. These conditions include mesenteric cyst, ${ }^{16}$ lymphoma (especially of the non-Hodgkin's variety), ${ }^{17}$ metastatic disease, carcinoid tumor and retractile mesenteritis, ${ }^{18}$ mesenteric desmoid tumor, ${ }^{1}$ lipoma, ${ }^{19}$ liposarcoma,${ }^{20}$ and Crohn's disease. Of these processes, only desmoid tumors have been associated with pregnancy.

CT can be of great value in assessing the size, location of origin, attenuation value, and the effect of a mesenteric mass on contiguous organs. ${ }^{1,3,4}$ In some cases, it can suggest the correct diagnosis, although the findings usually are not specific. When combined with clinical information, correct diagnosis is facilitated ${ }^{3}$

Patients with mesenteric lymphoma, unlike those with desmoid tumor, may have coexisting retroperitoneal lymphadenopathy. ${ }^{16}$ It has been stated that 61 percent of non-Hodgkin's lymphomas have mesenteric involvement at the time of presentation, whereas only 5 percent of patients with Hodgkin's lymphoma will have involvement of the mesentery. ${ }^{16}$ The "sandwich sign," as originally described by Mueller and coauthors, ${ }^{17}$ can be seen on sonography and CT when there is encasement of the mesenteric vessels and perivascular fat by adjacent mesenteric lymphomatous masses. However, a similar appearance was also reported by Baron and $\mathrm{Lee}^{1}$ in their two cases of mesenteric desmoid tumor. Thus, although the sandwich sign is strongly suggestive of mesenteric lymphoma, it cannot be considered diagnostic.

Patients with retractile mesenteritis and ileal carcinoid tumor typically demonstrate a well-defined mass in the right lower quadrant. The mass, which is of low density, has a stellate or a concentrated pattern of mesenteric neurovascular bundles. ${ }^{18}$ However, only patients with carcinoid will have an elevated 5-hydroxyindoleacetic acid level.

Lipoma can be diagnosed if a sharply marginated, homogeneous mass with a capsule and having a CT number equal to or less than normal fat is seen. ${ }^{20}$ However, it is important to realize that hemorrhage into a mass composed of fat can obscure the fat component, giving an overall appearance of solid tissue density.

Inflammatory diseases should also be considered in the differential diagnosis. It is well known that inflammatory changes can occur in the transverse mesocolon secondary to pancreatitis. Crohn's disease can cause thickening of the mesentery secondarily by formation of microabscesses from extension of sinus tracts into the mesentery or di- rectly by extension of the transmural inflammation. ${ }^{2}$ However, in the present case, there was no reason to suspect either of these diseases from the patient's history or laboratory values.

\section{Comment}

The case reported here represents a rare entity, mesenteric desmoid tumor, as a cause of gastric outlet obstruction in a pregnant patient. Ultrasonography demonstrated the mass, but CT gave a more explicit description of the location, density, and effect on contiguous organs. It was thought that angiography would not have contributed any vital information in this case. This conclusion is in agreement with the experience of others. ${ }^{18}$ Certainly, an upper gastrointestinal series and barium enema would have demonstrated abnormalities, but it is doubtful that they would have provided adequately specific information to forego further evaluation by CT. Whereas an upper gastrointestinal series would have shown compression of the gastric lumen and outlet obstruction, CT demonstrated the extraluminal pathologic alterations, thus providing important information.

Clearly, CT has come to assume a central role in the work up of patients with suspected primary or secondary mesenteric pathology. It is also an important modality for any patient in whom the disease may have a significant component that is extrinsic to gastrointestinal lumen.

1. Baron, R.L., and Lee, JKT: Mesenteric desmoid tumors. Sonographic and computed tomographic appearance. Radiology 140:777-9, Sep 81

2. Moss, A.A., Gamsu, G., and Genant, H.K.: Computed tomography of the body. W.B. Saunders Co., Philadelphia, 1983

3. Carr, L.Y., et al.: Gardner's syndrome and mesenteric desmoids. Am J Gastroenterol 80:310-2, Apr 85

4. Magid, D., et al.: Desmoid tumors in Gardner's syndrome. Use of computed tomography. AJR 142:1141-5, Jun 84

5. Pearman, R.O., and Mayo, C.W.: Desmoid tumors. A clinical and pathologic study. Ann Surg 115:114-25, Jan 42

6. Reitamo, J.J., et al.: The desmoid tumor. I. Incidence, sex-, age- and anatomical distribution in the Finnish population. Am J Clin Path 77:665-73, Jun 82

7. Brasfield, R.D., and Das Gupta, T.K.: Cited by op. cit., ref. 1

8. Simpson, R.D., Harrison, E.G., Jr., and Mayo, C.W.: Mesenteric fibromatosis in familial polyposis. A variant of Gardner's syndrome. Cancer 17:526-34, Apr 64

9. Karakousis, C.P., et al:: Mesenteric fibromatosis in Gardner's syndrome. Arch Surg 113:998-1000, Aug 78

10. Suarez, V., and Hall, C.: Mesenteric fibromatosis. Br J Surg 72:976-8, Dec 85

11. Häyry, P., et al.: The desmoid tumor. II. Analysis of factors possibly contributing to the etiology and growth behavior. Am J Clin Path 77:674-80, Jun 82

12. Lipschütz, A.: Cited by Svanvik, Jr., et al.: Desmoid tumor in the abdominal wall after treatment with high dose estradiol for prostatic cancer. Acta Chir Scand 148:301-3, 1982

13. Jadrijvic, D., Mardones, E., and Lipschūtz, A.: Antifibromatogenic activity of 19 nor- $\alpha$-ethinyltestosterone in the guinea pig. Proc Soc Exper Biol Med 91:38-9, Jan 56

14. Häyry, P., et. al.: The desmoid tumor. III. A biochemical and genetic analysis. Am J Clin Path 77:681-5, Jun 82 
15. Caldwell, E.H.: Desmoid tumor. Musculoaponeurotic fibrosis of the abdominal wall. Surgery 79:104-6, Jan 76

16. Bernardino, M.E., Jing, B.S., and Wallace, S.: Computed tomography diagnosis of mesenteric masses. AJR 132:33-6, Jan 79

17. Mueller, P.R., et al.: Appearance of lymphomatous involvement of the mesentery by ultrasonography and body computed tomography. The "sandwich sign." Radiology 134:467-73, Feb 80

18. Siegel, R.S., et al.: Computed tomography and angiography in ilial carcinoid tumor and retractile mesenteritis. Radiology 134:437-40, Feb 80

19. Heiken, J.P., Forde, K.A., and Gold, R.P.: Computed tomography as a definitive method for diagnosing gastrointestinal lipomas. Radiology 142:409-14, Feb 82

20. Friedman, A.C., et al: Computed tomography of abdominal fatty masses. Radiology 139:415-29, May 81
Accepted for publication in June 1985. Updating, as necessary, has been done by the authors.

At the time this paper was written, Dr. Dunn was a radiology resident at Kennedy Memorial Hospitals-University Medical Center, Stratford, New Jersey, where Paul J. Chase, D.O., is the radiology residency program director. Dr. Dunn is presently an attending radiologist at Delaware Valley Medical Center, Langhorne, Pennsylvania. Dr. Golkow is an attending radiologist and medical director of CT Scanning at KMH-UMC, Stratford, New Jersey.

Dr. Dunn, Delaware Valley Medical Center, Department of Radiology, 200 Oxford Valley Road, Langhorne, Pennsylvania 19047. 